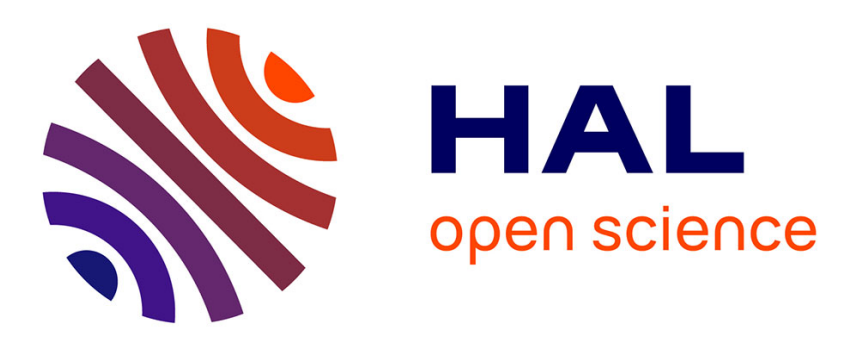

\title{
Assessing the Social Criteria for Human-Robot Collaborative Navigation: A Comparison of Human-Aware Navigation Planners
}

Harmish Khambhaita, Rachid Alami

\section{- To cite this version:}

Harmish Khambhaita, Rachid Alami. Assessing the Social Criteria for Human-Robot Collaborative Navigation: A Comparison of Human-Aware Navigation Planners. Proc. IEEE International Symposium on Robot and Human Interactive Communication (RO-MAN), Aug 2017, Lisbonne, Portugal. 6p. hal-01568841

\author{
HAL Id: hal-01568841 \\ https://hal.laas.fr/hal-01568841
}

Submitted on 25 Jul 2017

HAL is a multi-disciplinary open access archive for the deposit and dissemination of scientific research documents, whether they are published or not. The documents may come from teaching and research institutions in France or abroad, or from public or private research centers.
L'archive ouverte pluridisciplinaire HAL, est destinée au dépôt et à la diffusion de documents scientifiques de niveau recherche, publiés ou non, émanant des établissements d'enseignement et de recherche français ou étrangers, des laboratoires publics ou privés. 


\title{
Assessing the Social Criteria for Human-Robot Collaborative Navigation: A Comparison of Human-Aware Navigation Planners
}

\author{
Harmish Khambhaita $^{1}$ and Rachid Alami ${ }^{1}$
}

\begin{abstract}
This paper focuses on requirements for effective human robot collaboration in interactive navigation scenarios. We designed several use-cases where humans and robot had to move in the same environment that resemble canonical path-crossing situations. These use-cases include open as well as constrained spaces. Three different state-of-the-art humanaware navigation planners were used for planning the robot paths during all selected use-cases. We compare results of simulation experiments with these human-aware planners in terms of quality of generated trajectories together with discussion on capabilities and limitations of the planners. The results show that the human-robot collaborative planner [1] performs better in everyday path-crossing configurations. This suggests that the criteria used by the human-robot collaborative planner (safety, time-to-collision, directional-costs) are possible good measures for designing acceptable human-aware navigation planners. Consequently, we analyze the effects of these social criteria and draw perspectives on future evolution of human-aware navigation planning methods.
\end{abstract}

\section{INTRODUCTION}

We are witnessing a surge in social robots that are present in our everyday lives. Robots are offering guidance to passengers at airports [2], providing assistance to elderly people at care centers [3], or even engaging in entertaining experiences in public spaces [4]. Use of social robots in these domains show that situations where human and robot share and navigate in common space are becoming more and more important. An even higher degree of collaboration is necessary for fluent co-navigation, especially in confined spaces such as narrow corridors where human and robot have to act cooperatively and help each-other for finding their way.

Although there are several human-aware navigation planners proposed in the literature, to the best of our knowledge, no substantive approach yet proposed to compare and evaluate the planning schemes. In this paper we will consider several normative co-navigation situations as basis for comparing the performance of human-aware navigation planners. Therefore, we will refrain from going in to the implementation details (planning algorithms, robot control) and rather focus on assessment of paths generated by the planners and behavior of the robot during a full interactive navigation episode. Fig. 1 shows the situations we have designed in a simulated environment for evaluating the planners. From our experiments with multiple planners in different situations we have learned that parameter tuning

${ }^{1}$ LAAS-CNRS, Université de Toulouse, CNRS, Toulouse, France. \{harmish.khambhaita, rachid.alami\}@laas.fr

This work is supported by the European Union's Horizon 2020 research and innovation programme under grant agreement No. 688147 (MuMMER project).

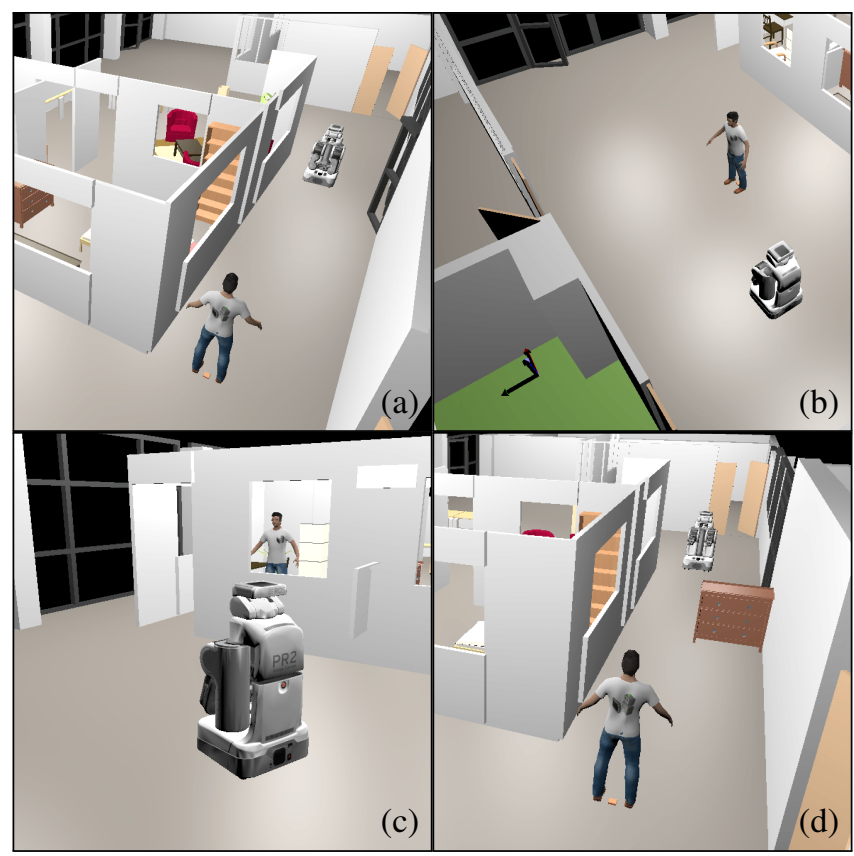

Fig. 1: Canonical human-robot path crossing situations. (a) Human and robot crossing each other's path on a corridor. (b) $90^{\circ}$ path crossing situation. (c) Human coming out of a room where robot needs to go, they cross each-other at the door. (d) Similar to situation (a), however the drawer is blocking the corridor in such a way that only one person (or robot) can pass next to the drawer at a time.

for particular situations is essential for any planner to show its full potential in solving the co-navigation situation.

\section{RELATED WORK}

A. Social cost based human-aware planning: Safe motion with respect to co-existing humans is the foremost requirement for social robots. Consequently some of the earlier work in human-aware navigation mainly concentrates on generating paths that keep a safe distance from humans, mainly by adopting the theory of proxemics [5]. The humanaware navigation planner introduced by [6] uses an algorithm that generates social costs in a grid-map structure around humans to facilitate $A^{*}$-like search algorithms to find paths that minimize such social costs. Furthermore, the authors have proposed other social criteria like visibility: it is better for the robot to make itself visible to the human for most part of their trajectory, and hidden zones: robot should not make itself appear very near from behind the human which 


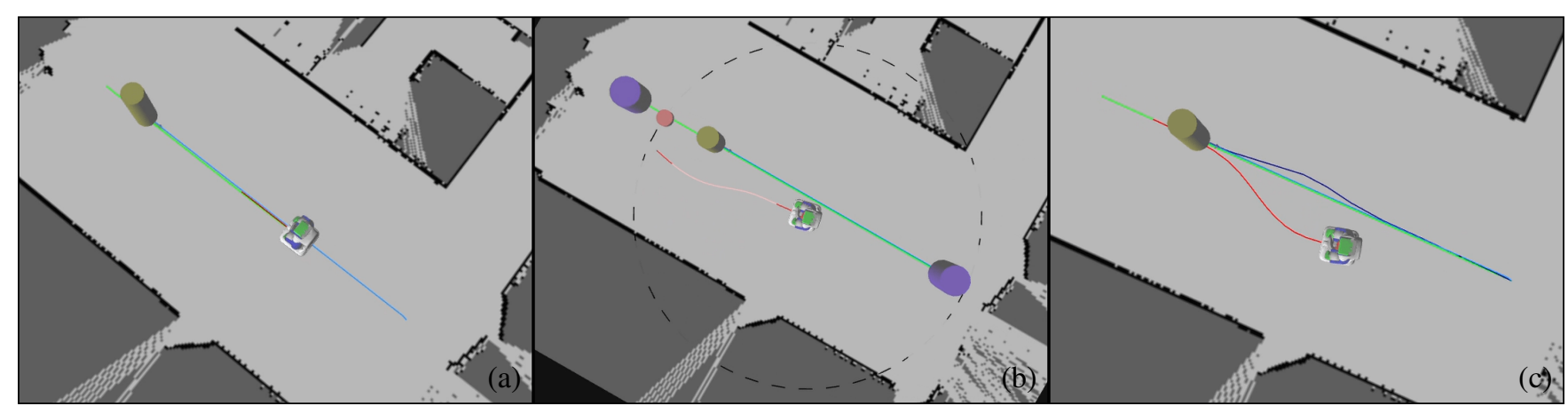

Fig. 2: Trajectories generated by human-aware planners for indoor hall navigation task. (a): Directional-cost planner. (b): AKP planner. (c): Cooperative planner. The robot and human are crossing each other's path in a face-to-face configuration. Since the area is relatively wide, the robot has enough latitude to move away from the predicted human path.

can shock humans. Recent surveys [7] [8] provide thorough analysis on the use of proxemics and other social costs in human-aware navigation planning.

One important aspect for co-operative motion is legibility, which enables an observer to quickly infer the robot intent and goals [9]. The directional cost model introduced by [10] has shown to increase legibility of the robot motions, where a robot attempts to solve a spatial conflict by adjusting velocity instead of the path when possible. From an extensive userstudy, it is clear that humans prefer robot following this strategy, particularly in $90^{\circ}$ path crossing situations [11]. In our comparison of human-aware planners, the first planner we have considered implements this strategy.

Another acclaimed approach used by the human-aware robotics researchers is the social force model [12]: a method to describe crowd dynamics. The robot navigation planners explained in [13] and [14] use the social force model to cope with uncertain human motions. In the extended social force model [15], [16] approach, every iteration of planning step uses the human prediction information which is dependent on the path calculated during the previous iteration. This is the second planner we are using for our comparison. The planning schemes based on the social force model works nicely in large or open spaces where the robot have enough latitude to move away from predicted human paths. However, as we will see, it performs rather poorly in constrained situations. An important concept brought in by these methods is to interlace prediction of human motions within the robot navigation planning framework. Because of superior results, we believe that any further human-aware navigation planner should also involve prediction of human motions in their planning architecture.

B. A case for human-robot cooperative planning: An elastic band [17] augmented with temporal information for optimizing robot trajectories is introduced in [18]. In this approach, the optimization framework locally deforms the robot trajectory that includes a series of time-difference values between each successive poses, instead of deforming a purely geometric path. The resulting timed elastic band makes it easy to take kinodynamic and nonholonomic constraints into account, formalizing the optimization problems as a nonlinear least squares problem.

We have substantially extended this work by introducing prediction and optimization of human trajectories within the same framework [1]. In this planning scheme the robot uses the same environmental map to coherently plan its own trajectory and predict plausible human trajectories, therefore it always provides a solution where both human and robot can move optimally. The co-navigation solution does not include only the contributions of the robot but also of the human, and that is why we claim that it is a human-robot cooperative planner. However, human and robot are not treated equally in this scheme, generally the robot takes, when possible, all or most of the effort to avoid colliding with humans. With this planner the robot not only can react to the unfolding situation but it can also proactively suggest a solution to the co-navigation situation, especially in confined spaces. In this approach, our focus is on advanced interactive motions from a single person to a small group of people. For open spaces, this cooperative planner gives comparable results to the social force model based approach. However, in confined spaces it give arguably better results by eliminating unnecessary detours and remaining adaptive to the human motions. This is the third planner we are using for comparison of human-aware navigation planners discussed in the following section.

\section{COMPARING HUMAN-AWARE PLANNERS}

We have compared three human-aware navigation planner in five customary human-robot path crossing situations. All of the planners utilize the well-known robot navigation architecture move_base [19], developed as a local planner plug-ins to it ${ }^{1}$. We have constructed a simulated environment with MORSE ${ }^{2}$ that resembles the real robotics lab at LAASCNRS. Fig. 1 shows screen shots of the simulator and situations we have designed for testing the planners. We have used a simulated version of the PR2 robot for these

\footnotetext{
${ }^{1}$ Directional-cost: http: // harmish. in/HANP / code/planner

AKP: https://devel.iri.upc.edu/pub/labrobotica

Cooperative: http: //harmish.in/Coop/code

${ }^{2}$ https: / / www. openrobots.org/morse
} 


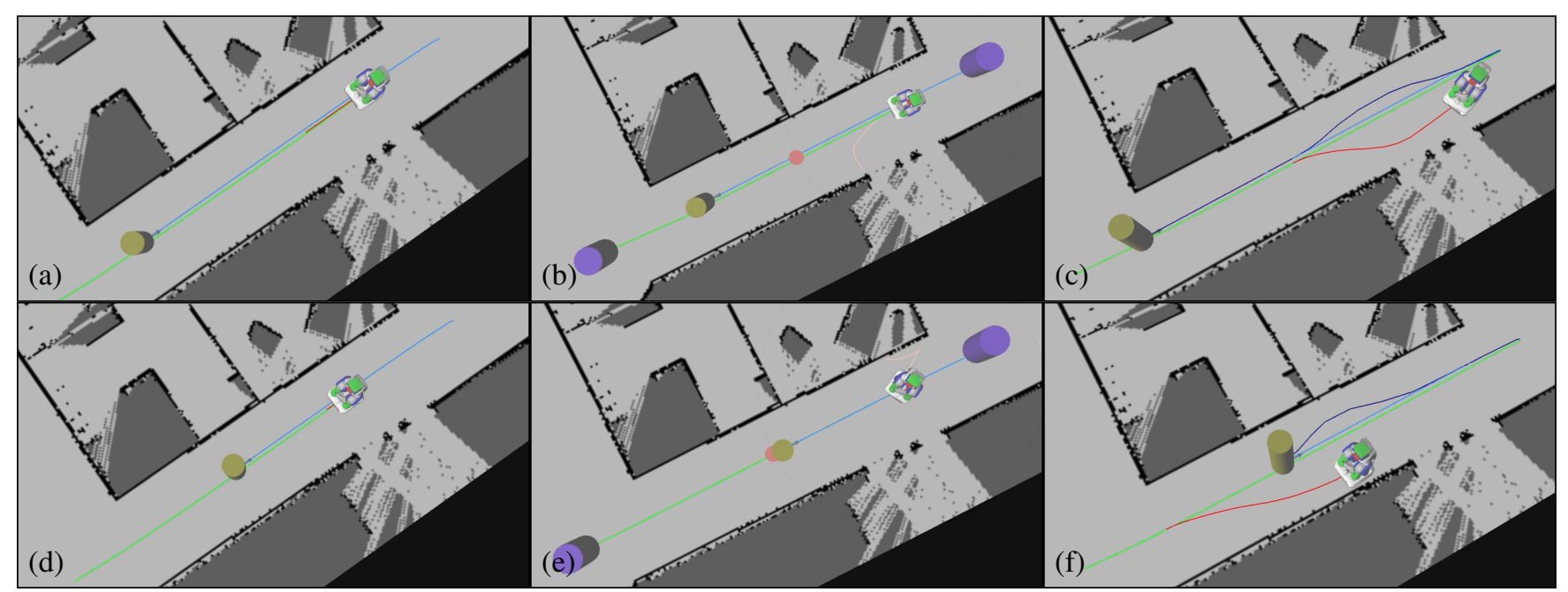

Fig. 3: Trajectories generated by human-aware planners for corridor crossing task. (a) and (d): Directional-cost planner. (b) and (e): AKP planner. (c) and (f): Cooperative planner. Figures on the top row shows snapshots of situations that happen a few seconds before the situations shown in the bottom row. Here also the robot and human are crossing each other's path in a face-to-face configuration, however, in a confined space.

experiments. An advantage of simulation is that it provides a consistent and reproducible environment for testing different navigation algorithms. For simulating human motions, we have developed a human navigation package, based on the design of the move_base framework, which can simultaneously simulate the motion of multiple humans. This simulator uses an $A^{*}$-like algorithm for planning global paths and a teleportation controller as local planner, which simply moves the humans on the global path at nominal walking velocity ${ }^{3}$.

Thus, for each situation we give the exact same start and goal positions of the robot and human for all three planners. As a shorthand we will use the names directionalcost planner for the planner in [11], AKP planner for planner in [16] and cooperative planner for planner in [1]. All of the comparison figures show both global (in green) and local (in red) paths of the robot. Whenever possible the predicted human paths are also shown in the figures (in blue) ${ }^{4}$.

A. Indoor hall navigation: In the first situation we have considered a hall size area where robot and human are crossing each other's path in a face-to-face configuration. Fig. 2 shows a particular scene during the navigation task for each planner. Here trajectories generated by both AKP and cooperative planners make the robot move away from the predicted human path thus keeping a comfortable distance from the human and require minimal or no effort from the human in the collision avoidance task. Since, the directionalcost planner only plans in velocity, it slows down as the human approaches near and eventually stops completely. Once the human moves behind the robot the robot continues on its path, thus it requires the human to go around the robot. The directional-cost based planner relies fully on the global

\footnotetext{
${ }^{3}$ http: //harmish.in/HSIM/code

${ }^{4}$ The purple cylinder shown in the AKP planner examples are the goal positions used for the human goal prediction.
}

planner to generate paths. Thus, if we use the global planner with a continuous re-planning scheme in case when the human stops in front of the robot, the robot will eventually change its direction as well, as soon as a new global plan is available. However, here we are purely comparing the local planner, so to remain fair to the other two planner we are not using the re-planning mechanism for the global planner.

B. Corridor crossing: Second situation is quite common in the office, airport, or shopping mall like environments. Here the human and the robot are crossing their paths similar to the previous situation, however, since the passage is narrow it requires some effort from both human and robot to avoid a collision (see fig. 3). In case of the directionalcost planner the robot again simply slows down as the human approaches, the local plan of the robot gets smaller. Thus, even this behavior is understandable by the human, it requires human to go around the robot.

The AKP planner starts properly on its path, however, as the human comes nearby it plans a trajectory backwards. Such back-tracking is common in social force model based planners. A possible explanation for this behavior would be: while the repulsive force of the walls remains same on both sides of the robot, as human approaches the repulsive force from human gets larger than the attractive force towards the robot's goal, which eventually results into the robot moving backwards 5 . As we can see in the figure $3 \mathrm{c}$, the cooperative planner moves on the one side of the corridor well ahead in time. It does so because it has predicted a human path in the same map that requires least effort from the human in terms of changing their current path. It should be noted that, in this case it is necessary for both human and robot to change

\footnotetext{
${ }^{5}$ Because of backtracking and due to the design of AKP planner, only for this planner we had to add an imaginary laser scanner on the back of the PR2 robot in simulated environment
} 


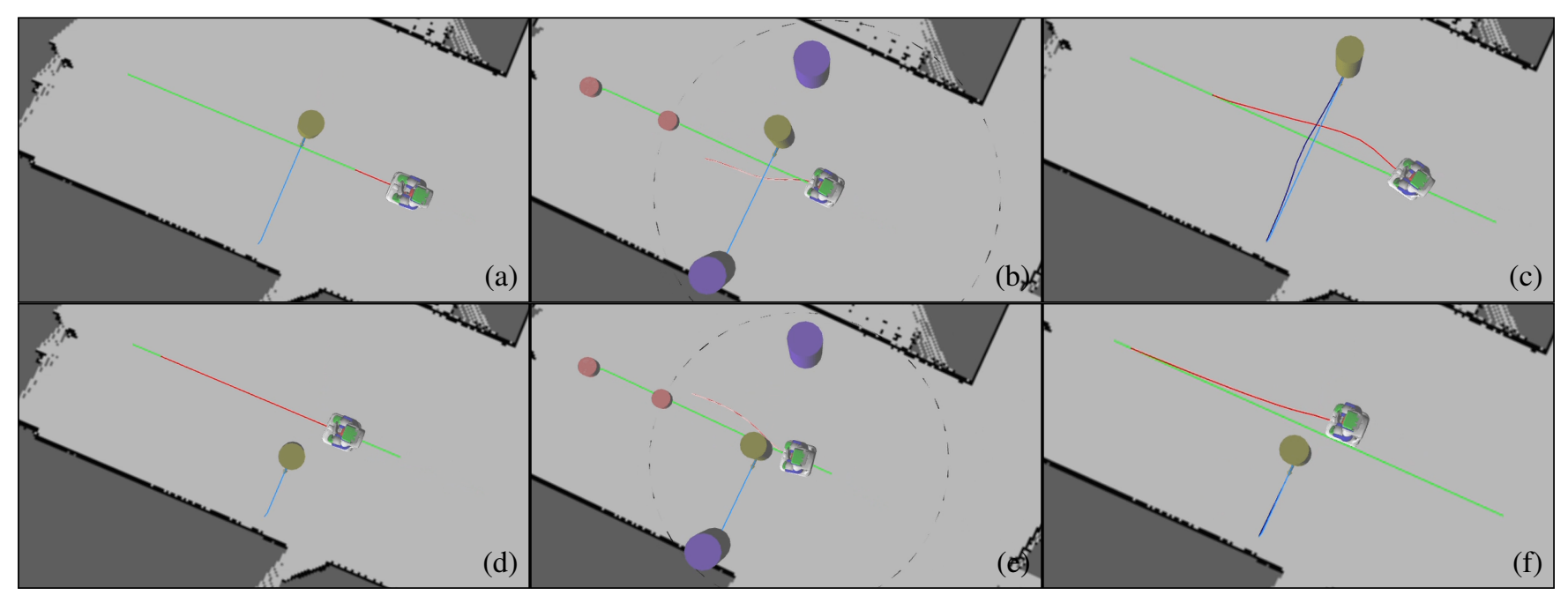

Fig. 4: Trajectories generated by human-aware planners for $90^{\circ}$ path-crossing task. (a) and (d): Directional-cost planner. (b) and (e): AKP planner. (c) and (f): Cooperative planner. Figures on the top row shows snapshots of situations that happen a few seconds before the situations shown in the bottom row.

their paths to find a solution to the co-navigation problem. Here the robot selects a solution that causes minimal change to the human trajectory.

C. $90^{\circ}$ path-Crossing: Fig. 4 shows a situation where robot and human are crossing their paths at a $90^{\circ}$ angle. Here the directional-cost planner performs quite good as the robot slows down when a human comes nearby, and then the robot accelerates again towards its goal, no additional effort required by the human here. It should be noted that the directional-cost planner was extensively tested on this situation, thus it generates paths that are legible and acceptable by the humans. AKP planner initially computes a path to cross in front of the human, but when the human is closer to the robot, the planner eventually decided to traverse behind the human. Here, we suspect this behavior is due to the particular workings of random exploration based algorithm used by the AKP planner. Nevertheless, $A^{*}$-like path search algorithm with continuous re-planning scheme also yields similar behavior.

The cooperative planner simultaneously changes its path while slowing down, thus suggesting the human to pass before itself. Since, we are using both directional and timeto-collision constraints for this planner, this can be explained as a combined effect of those social constraints. Therefore, the resulting behavior remains as legible as, or arguably even better than the directional-cost planner.

D. Passing through a door: As shown in figure 1c, often in a door-crossing situation the human is visible to the robot due to the window between the hall area and the room. Fig. 5 shows trajectories generated by the planners when the task of the robot is to move inside the room while the human is moving out. The directional-cost planner could induce "bad" configurations, where it reaches near the door and slows down but does not give enough space to the human to move out of the room. This could lead to considerable effort by the human, making them move back to create space for the robot. The AKP planner, because of its backtracking behavior could eventually make space for the human. However, as we can see in fig $5 \mathrm{~d}$ the robot goes very near to the door which could threaten the human. With the cooperative planner the robot prefers waiting in a place where it limits, as much as possible, obstruction to the human motion. Instead of moving backwards, here the robot proactively plans a path that is not only far from the door when human is coming out, but also the robot trajectory inherently contains a "waiting" behavior.

E. Constrained corridor crossing: The last situation we have considered for comparison is similar to the corridor crossing situation described in Sec. III-B, however with an additional obstacle in the corridor. Fig. 1d depicts this situation in the simulation environment. The corridor becomes a highly constrained space, where robot and human cannot even pass side-by-side. Only the human or the robot can cross the additional obstacle at a time, requiring the other to either wait or backtrack. The additional obstacle (a drawer) is not known to the robot, that is, it is not in its pre-built map. Therefore, we are also showing laser scanner points (in yellow) to better understand the robot behavior.

Here both directional-cost and AKP planner come very near to the obstacle at the same time when the human also reaching near it. Directional-cost planner hardly gives enough space for the human to pass, while AKP planner makes the robot move backwards when human comes nearby the robot. Since with both of these planners the robot slows down to almost standstill, they are not violating the safety requirement. However, with both planners the human needs to share most of the effort for avoiding a collision with the robot. The cooperative planner performs particularly well in this situation. As shown in the fig. 6c, the robot first moves near the drawer, and waits for the human to pass, and then continuous its motion towards its goal. 


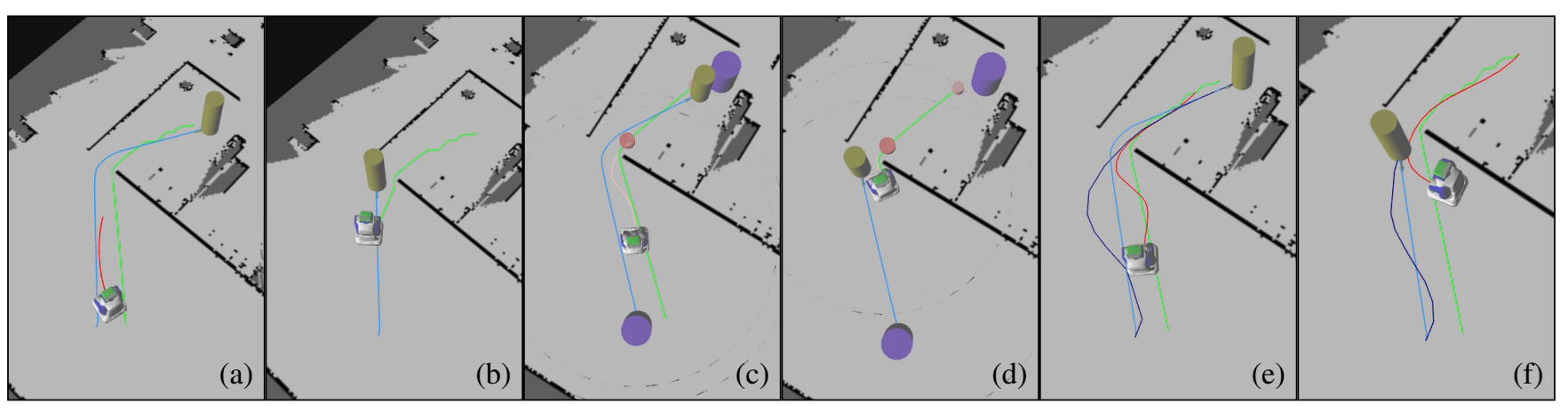

Fig. 5: Trajectories generated by human-aware planners for the robot passing through a door. (a) and (b): Directional-cost planner. (c) and (d): AKP planner. (e) and (f): Cooperative planner. Figures a, c and e shows snapshots of situations that happen a few seconds before the situations $b, d$ and $f$ respectively.

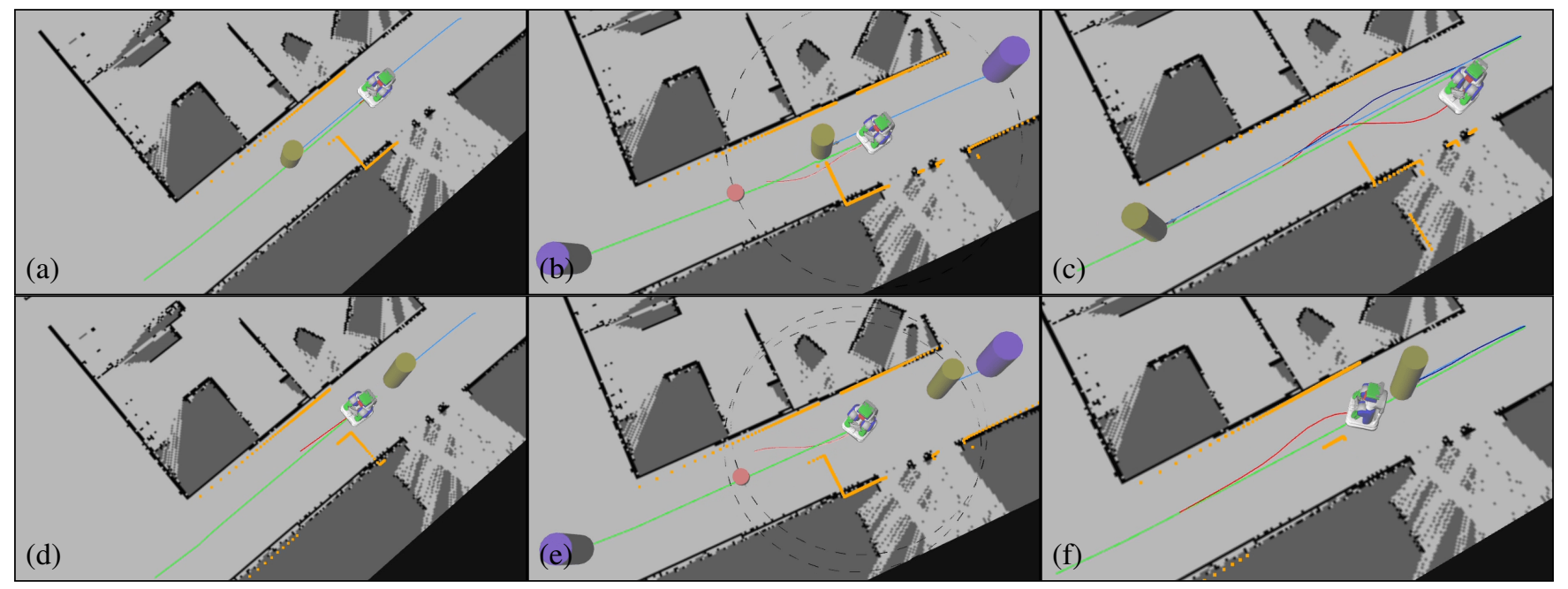

Fig. 6: Trajectories generated by human-aware planners for corridor crossing task with an obstacle in the middle of the hall. (a) and (d): Directional-cost planner. (b) and (e): AKP planner. (c) and (f): Cooperative planner. Figures on the top row shows snapshots of situations that happen a few seconds before the situations shown in the bottom row.

\section{DISCUSSION AND CONCLUSIONS}

We have shown capabilities and drawbacks of three different human-aware navigation planners. The cooperative planner performs well in both confined and non-confined cases. The behavior such as stopping near the door and facilitating human in confined corridors emerges due to the social constraints that are integrated in the optimization framework. Furthermore, the cooperative planner enables balancing and tuning of the efforts between the human and the robot to solve a co-navigation task. The inspiration for designing a tunable navigation planner comes from previously-proposed approaches for geometric [20] and symbolic [21] planning systems, where the robot synthesizes a shared plan for the human and itself. By tuning, we mean to adjust the "elasticity" of underlying timed elastic band. Fig. 7 shows the effect of tuning the effort between a human and a robot for a shared navigation task. The cooperative planner is also highly reactive at the same time, so if during a navigation task the human decides to move on a different path than the one suggested by the robot, the robot quickly adapts its path.

With all other parameters being equal, fig. 8 shows the effect of the safety constraint on the robot path. With a single parameter we can tune how far the robot moves from the human. The cooperative planner also exploits results of user study in [11] and introduce directional costs in the optimization framework. The directional costs discourages face-to-face motion and makes the robot slow down when human and robot move opposite to each other.

A novel constraint used by the cooperative planner is time-to-collision, defined as the projected time to a possible future collision with a human. Recent empirical studies of pedestrian interactions have shown that time-to-collision can uniquely describe how humans move in public places [22]. It is also interesting to note that, the same study also shows that the social force model rather poorly fits to the pedestrian interaction data, and thus being a weak model to design human-robot interactive navigation planners. Fig. 9 shows an example of how this time-to-collision constraint affects the generated path. When time-to-collision constraint is switched-on the robot moves early to its right signaling that it is already avoiding possible collision with the human, thus making the robot behavior proactive. 


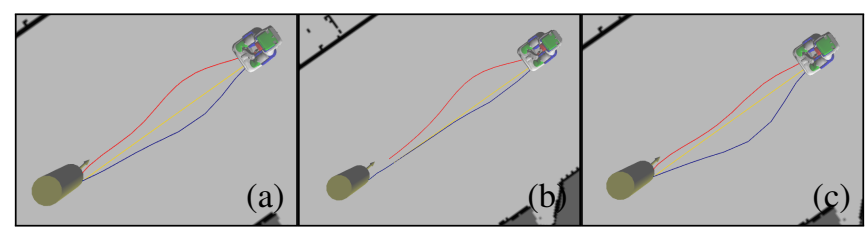

Fig. 7: Balancing the shared effort between human and robot. (a) Human and robot share equal effort. (b) The robot shares almost full effort and moves away from predicted human path. (c) Although never used in real world situations, it is possible to design a rude behavior of the robot where it expects human to make more effort for avoiding a collision.

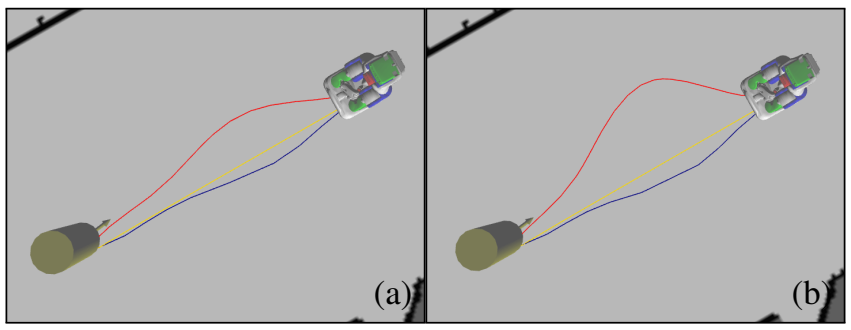

Fig. 8: Effect of the safety constraint on robot paths. (a) Minimum safety distance between human and robot is set to 0.3 meters. (b) Minimum safety distance is set to 0.7 meters.

We learned the following lessons from this comparison:

- Directional-cost planner performs well in $90^{\circ}$ path crossing and sub-optimally in constrained situations.

- Social force model based planners can perform well in open areas or large indoor environments, however they suffer from unnecessary detours due to the way repulsive and attractive forces are calculated.

- The cooperative planner performs well both in nonconstrained and constrained situations, it produces legible and cordial behaviors in confined areas.

- It is imperative for a human-aware navigation planner to tightly couple with human motion prediction method.

Although simulation is a limited tool, repeatability of the same experiments makes it a suitable tool for evaluating different navigation algorithms on the same situations. We believe that our investigation provides preliminary basis for comparing human-aware navigation planners. Drawing fruitful insights from these experiments, we think the cooperative planner is ready for extended evaluation on the real robot. Therefore, we are planning a comprehensive user-study to further evaluate the cooperative planner and compare it against other human-aware navigation planners.

\section{REFERENCES}

[1] H. Khambhaita and R. Alami, "A Human-Robot Cooperative Navigation Planner," in Proc. the Companion of the ACM/IEEE International Conference on Human-Robot Interaction (HRI), 2017.

[2] R. Triebel, K. Arras, et al., "SPENCER: A Socially Aware Service Robot for Passenger Guidance and Help in Busy Airports," in Proc. Conference on Field and Service Robotics (FSR), 2016.

[3] D. Hebesberger, C. Dondrup, et al., "Lessons learned from the deployment of a long-term autonomous robot as companion in physical therapy for older adults with dementia - A Mixed Methods Study,” in

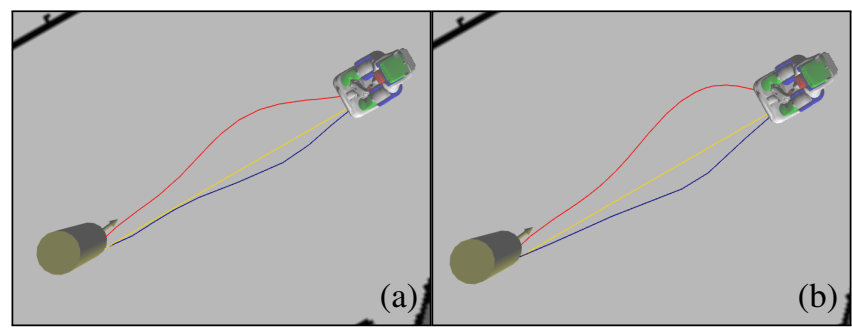

Fig. 9: Effect of the time-to-collision constraint on robot paths. (a) Without time-to-collision constraint. (b) time-tocollision constraint is switched-on.

Proc. ACM/IEEE International Conference on Human-Robot Interaction (HRI), 2016

[4] M. E. Foster, R. Alami, et al., "The MuMMER Project: Engaging Human-Robot Interaction in Real-World Public Spaces," in Proc. 8th International Conference on Social Robotics (ICSR), 2016.

[5] E. T. Hall, The Hidden Dimension: Man's Use of Space in Public and Private, 1966.

[6] E. A. Sisbot, L. F. Marin-Urias, et al., "A Human Aware Mobile Robot Motion Planner," IEEE Transactions on Robotics, vol. 23, no. 5, 2007.

[7] T. Kruse, A. K. Pandey, et al., "Human-aware robot navigation: A survey," Robotics and Autonomous Systems, vol. 61, no. 12, 2013.

[8] J. A. Rios Martinez, A. Spalanzani, and C. Laugier, "From Proxemics Theory to Socially-Aware Navigation: A Survey," International Journal of Social Robotics, vol. 7, no. 2, 2014.

[9] A. D. Dragan and S. Srinivasa, "Generating Legible Motion," in Proc. Robotics: Science and Systems, June 24 - June 28, 2013.

[10] T. Kruse, P. Basili, et al., "Legible Robot Navigation in the Proximity of Moving Humans," in Proc. IEEE Workshop on Advanced Robotics and Its Social Impacts (ARSO), 2012.

[11] T. Kruse, A. Kirsch, et al., "Evaluating Directional Cost Models in Navigation," in Proc. ACM/IEEE International Conference on HumanRobot Interaction (HRI), 2014.

[12] D. Helbing and P. Molnár, "Social force model for pedestrian dynamics," Physical Review E, vol. 51, no. 5, 1995.

[13] G. Ferrer, A. Garrell, and A. Sanfeliu, "Robot companion: A socialforce based approach with human awareness-navigation in crowded environments," in Proc. IEEE/RSJ International Conference on Intelligent Robots and Systems (IROS), 2013.

[14] M. Kuderer, H. Kretzschmar, et al., "Feature-Based Prediction of Trajectories for Socially Compliant Navigation," in Proc. Robotics: Science and Systems (RSS), July 9-13, 2012.

[15] G. Ferrer and A. Sanfeliu, "Proactive Kinodynamic Planning using the Extended Social Force Model and Human Motion Prediction in Urban Environments," in Proc. IEEE/RSJ International Conference on Intelligent Robots and Systems (IROS), 2014.

[16] G. Ferrer and A. Sanfeliu, "Multi-Objective Cost-to-Go Functions on Robot Navigation in Dynamic Environments," in Proc. IEEE/RSJ International Conference on Intelligent Robots and Systems (IROS), 2015.

[17] S. Quinlan and O. Khatib, "Elastic Bands: Connecting Path Planning and Control," in Proc. IEEE International Conference on Robotics and Automation (ICRA), 1993.

[18] C. Rösmann, W. Feiten, et al., "Trajectory modification considering dynamic constraints of autonomous robots," in Proc. German Conference on Robotics (ROBOTIK), 2012.

[19] E. Marder-Eppstein, "move_base: A ROS package that lets you move a robot to desired positions using the navigation stack."

[20] J. Waldhart, M. Gharbi, and R. Alami, "Planning handovers involving humans and robots in constrained environment," in Proc. IEEE/RSJ International Conference on Intelligent Robots and Systems (IROS), September 28 - October 2, 2015.

[21] J. Shah, J. Wiken, et al., "Improved Human-Robot Team Performance Using Chaski, A Human-Inspired Plan Execution System," in Proc. ACM/IEEE International Conference on Human-Robot Interaction (HRI), March 6-9, 2011.

[22] I. Karamouzas, B. Skinner, and S. J. Guy, "Universal Power Law Governing Pedestrian Interactions," Physical Review Letters, vol. 113, no. 23, 2014. 